\title{
Adapted Physical Activity Interventions among the Elderly: Their Effects on Physical Performance
}

\author{
Silvia Coppola, Francesca D'Elia, Rodolfo Vastola and Maurizio Sibilio \\ Department of Human, Philosophical and Educational Sciences, University of Salerno, Fisciano Salerno 84084, Italy
}

\begin{abstract}
This study is part of a research project on testing the effectiveness of an APA (adapted physical activity) protocol aimed at promoting the maintenance and development of autonomy and wellbeing among the elderly. The research was carried out in two elderly people centers located in Salerno (Southern Italy). The sample consisted of 33 people aged between 69 and 97 years (81.53 \pm 7.02). The experimental group consisted of 18 participants aged between 70 and $92(81.11 \pm 6.85)$, while the control group of 15 participants were between 69 and 97 years old $(82 \pm 7.43)$. The effects of the APA protocol, was measured using the SPPB (Short Physical Performance Battery) before and after the 18-week intervention, which included two weekly sessions of structured training modules focusing on the coordinating and organic conditioning of the elderly. The results obtained found a substantial improvement in motor performance in the experimental group compared to the control group (72.2\% vs. 33.3\%) and hence suggest the need for further research on the effects of APA interventions, across a larger range of subjects.
\end{abstract}

Key words: Adapted physical activity, active aging, SPPB, elderly.

\section{Introduction}

Ageing is associated with a general decline of motor skills, which is manifested by a reduction in muscle strength, joint mobility, difficulty maintaining balance and increased risk of falls [1-3]. An essential factor for the promotion and maintenance of good health in the elderly population, constantly highlighted both in European and global policies $[4,5]$, is the practice of physical activity $[6,7]$. An enabling environment for the promotion and diffusion of adapted physical activity are the homes and day care facilities for the elderly [8-11]. According to the last census conducted in 2013 by the Ministry of Home Affairs, the number of registered residential structures in Italy was 6,467, accommodating a total of 303,180 elderly residents. Added to these are 7,717 day centers who welcome 900,000 elderly people; resulting in an average of 118 elderly people per center [12].

The structuring of a specific physical activity program for the elderly is proposed as a tool for

Corresponding author: Maurizio Sibilio, professor, research fields: special didactic and pedagogy. primary and secondary prevention of diseases, and aims to counteract the damage caused by the physiological decline of psychomotor function in the elderly [13-16]. The objectives of the proposed APA (adapted physical activity) program for the elderly are the improvement, maintenance and recovery of the global residual potential of the elderly, with particular reference to the improvement of the ability of static and dynamic balance, the increase in muscle strength, the improvement of joint mobility and of gait functioning and coordination [17-19]. To assess the elderly's motor performanace (gait speed, balance, and the strength of the lower limbs) was used the Short Physical Performance Battery (SPPB) [20-21], developed by the National Institute on Aging. This is a validated battery specifically designed for the third age, with the aim of investigating the motor performance among the elderly [20-24].

\section{Objective}

The objective of this study was to evaluate possible improvements and/or consolidations of motor performance (gait speed, balance, and the strength of 
the lower limbs) as a result of the implementation of structured APA (adapted physical activity)through the use of the SPPB.

\section{Methodology}

\subsection{Study Design}

This study was designed as a prospective controlled study with a five-month follow-up.

\subsection{Participants}

The research was carried out in two centers for the elderly, one residential home and one day centre, located in Salerno (southern Italy). The sample consisted of 33 elderly (11 men and 22 women) aged between 69 and 97 years $(81.53 \pm 7.02)$. The experimental group consisted of 18 participants (six men and 12 women) aged between 70 and 92 years (81.11 \pm 6.85$)$, while the control group was composed of 15 elderly people (5 men and 10 women) aged between 69 and 97 years $(82 \pm 7.43)$. The two groups were homogeneous with respect to age and sex distribution.

\subsection{Instruments}

The SPPB (Short Physical Performance Battery) [25] was administered before and after the 18-week adapted physical activity intervention.

The SPPB is a standardized group of measures specifically designed to combine the results of the gait speed, balance, and the strength of the lower limbs among the elderly. The SPPB involves three timed performance tests: (1) a balance test, which requires the elderly to stand in equilibrium for up to 10 seconds and a total of 30 seconds in three different positions of increasing difficulty: feet together, in semi-tandem (side of the heel of one foot touching the big toe of the other foot) and in full tandem (feet directly in front of each other); (2) gait test which involves running for 4 meters at normal walking speed; (3) sit to stand test, which measures the time taken for the individual to sit down and stand from a chair with arms folded across the chest for 5 times in a row [25].

The total score ranges from 0 to 12 , and it is possible to classify individuals into three categories: seriously compromised in motor ability (score from 0 to 4), moderately compromised (score from 5 to 8 ) and in good condition (score greater than 8).

\subsection{The APA Protocol}

The structured physical activity intervention, was spread over a period of five months with meetings held twice a week. The adapted physical activity intervention was structured in 40-50-minute sessions and consisted of specific exercises aimed at increasing muscle strength, flexibility, balance and coordination. The physical activity routine was structured according to the principle of progression, starting from low-intensity exercises until reaching moderately-intense levels of activity. Initially, a careful analysis of the level of autonomy and physical efficiency was carried out by asking the individuals to perform exercises of low cardio-vascular and coordinative commitment (walking, marching in place, simple coordination exercises, static and dynamic balance, etc.).

The Protocol consisted of three main phases:

3.4.1 Initial Neuromuscular Activation Phase

The activity protocol included an initial neuromuscular activation phase primarily consisting in ambulation exercises. The walking phase was carried out in a progressive manner in terms of time and intensity. In cases where the individuals did not show sufficient stability, the activity was carried out with the assistance of an operator. In this case walking was a means to stimulate the elderly to improve balance and coordination. At this stage the activity did not envisage further combined movements.

Two weeks after the commencement of the intervention, following two minutes of walking, arm movements and some other light movements were simultaneously introduced. The exercises introduced at later stages of the intervention in this phase (gaits, 
marching, etc.) aimed at improving the capacity of dynamic balance and increase strength and coordination of the lower limbs in order to promote the prevention of falls.

\subsubsection{Central Phase}

The middle phase of the APA protocol included exercises for muscle strengthening, joint mobility, coordination and balance, done almost exclusively by sitting or with the help of the chair as a support for balance.

Before starting this phase of the routine, posture while sitting was corrected: back straight, angle between thigh and lower leg at $90^{\circ}$ and legs slightly apart.

As in the initial phase, the coordination exercises were proposed in a simple and slow manner, but were then progressively integrated with combined and asymmetric exercises.

With the same principle of graduality in mind, in the first phase of muscle strengthening exercises a single set of eight executions was proposed and, in later stages, these were extended to two sets of eight. The exercises were as follows: (1) arms positioned backwards in the sagittal plane in intra and extra-rotation (for the tonic postural muscles); (2) back position of the shoulders (for the tonic postural muscles); (3) abduction and adduction of the arms (for the shoulder muscles and tonic postural); (4) flexion, extension and abduction of the thigh (to strengthen the muscles of the lower limb); (5) squats performed from a sitting position with the aid of a chair (for the muscles of the lower limbs).

With regard to joint mobility the following mobilization exercises were proposed: (1) cervical spine exercises with flexions, inclinations and rotations of the head; (2) side inclinations for the trunk; (3) elevations, depressions, anteposition and retroposition of the shoulders; (4) flexion, extension, abduction and adduction of the wrists and hands; (5) bending, extension, abduction and adduction of the fingers and hands; (6) dorsal flexion, plantar flexion, inversion and subversions of the feet.

\subsubsection{Cool-down Phase}

The cool-down, programmed according to the intensity of the activity performed in the middle phase, aimed at restoring the body to the baseline. Walking combined with breathing exercises is considered particularly favorable to reach this aim with the elderly.

\subsection{Data Analysis}

The data were analyzed using descriptive statistics in order to trace the relevant parameters (age and sex distribution of the sample; changes in SPPB score from baseline classified into three categories: improvement, unchanged and worsening). Data are reported as mean \pm standard deviation or as absolute number with the percentage. Statistical analyses were performed using Microsoft Office Excel 97-2003. Changes in SPPB score from baseline to follow-up were classified under three categories (improvement of at least 0.1 point, unchanged and worsening).

\section{Results}

The sample consisted of 33 elderly, 22 women and 11 men, aged between 69 and 97 years. The average age was 81.53 years, with a SD 7.02. The majority of the sample (48\%) was in the 80-89 year range; followed by the age group between 70 and 79 years (39\%) (Table 1). The distribution for sex refers to the entire sample and was prevalently constituted by women with a ratio of 2:1.

The experimental group consisted of 18 elderly, 12 women and 6 men, aged between 70 and 92. The average age was 81.11 years, with a S.D. 6.85 (Table 2).

The majority of the sample (88\%) was in the 70-89 year range; followed by the age group over 90 years $(11 \%)$. The distribution for sex in the experimental group, as in the total sample, is constituted by a prevalence of women with a ratio of $2: 1$.

The control group consisted of 15 elderly, 10 women and 5 men, aged between 69 and 97 years. The average age amounted to 82.02 years, with a S.D. 7.43. 
Table 1 Age and sex distribution of the sample.

\begin{tabular}{|c|c|c|c|c|c|c|c|}
\hline \multicolumn{8}{|l|}{ Age class } \\
\hline Average & S.D. & Min. & Max. & \multirow[t]{2}{*}{$\mathrm{N}$} & \multirow[t]{2}{*}{ Freq. \% } & \multirow[t]{2}{*}{$\mathrm{N}^{\circ}$ men } & \multirow[t]{2}{*}{$\mathrm{N}^{\circ}$ women } \\
\hline 81.5 & 7.02 & 69 & 97 & & & & \\
\hline$\leq 69$ years & & & & 1 & $3 \%$ & 0 & 1 \\
\hline $70-79$ year & & & & 13 & $39 \%$ & 5 & 8 \\
\hline $80-89$ year & & & & 16 & $48 \%$ & 5 & 10 \\
\hline$\geq 90$ years & & & & 3 & $9 \%$ & 1 & 1 \\
\hline Total & & & & 33 & $100 \%$ & 11 & 22 \\
\hline
\end{tabular}

Table 2 Age and sex distribution of the experimental group.

\begin{tabular}{|c|c|c|c|c|c|c|c|}
\hline \multicolumn{8}{|l|}{ Age class } \\
\hline Average & S.D. & Min. & Max. & $\mathrm{N}$ & Freq. $\%$ & $\mathrm{~N}^{\circ}$ men & $\mathrm{N}^{\circ}$ women \\
\hline 81.11 & 6.85 & 70 & 92 & & & & \\
\hline$\leq 69$ years & & & & 0 & 0 & 0 & 0 \\
\hline $70-79$ years & & & & 8 & $44 \%$ & 3 & 5 \\
\hline $80-89$ years & & & & 8 & $44 \%$ & 3 & 5 \\
\hline$\geq 90$ years & & & & 2 & $11 \%$ & 0 & 2 \\
\hline Total & & & & 18 & $100 \%$ & 6 & 12 \\
\hline
\end{tabular}

Table 3 Age and sex distribution of the control group.

\begin{tabular}{|c|c|c|c|c|c|c|c|}
\hline \multicolumn{4}{|l|}{ Age class } & \multirow{3}{*}{$\mathrm{N}$. } & \multirow{3}{*}{ Freq. $\%$} & \multirow{3}{*}{$\mathrm{N}^{\circ}$ men } & \multirow{3}{*}{$\mathrm{N}^{\circ}$ women } \\
\hline Average & S.D. & Min. & Max. & & & & \\
\hline 82.02 & 7.43 & 69 & 97 & & & & \\
\hline$\leq 69$ years & & & & 1 & $7 \%$ & 0 & 1 \\
\hline $70-79$ years & & & & 5 & $33 \%$ & 2 & 3 \\
\hline $80-89$ years & & & & 7 & $46 \%$ & 2 & 5 \\
\hline$\geq 90$ years & & & & 2 & $13 \%$ & 1 & 1 \\
\hline Total & & & & 15 & $100 \%$ & 5 & 10 \\
\hline
\end{tabular}

Table 4 Changes in SPPB score from baseline classified into three categories (improvement, unchanged and worsening) in experimental group.

\begin{tabular}{lllll}
\hline Changes & Balance & Walk test & Sit to stand & Total \\
\hline Improvement & $50 \%$ & $27.7 \%$ & $38.8 \%$ & $72.2 \%$ \\
Unchanged & $50 \%$ & $55.5 \%$ & $55.5 \%$ & $16.6 \%$ \\
Worsening & 0 & $16.6 \%$ & $5.5 \%$ & $11.1 \%$ \\
\hline
\end{tabular}

The majority of the sample (46\%) are in the range 80-89 years; followed by the age group in the range 70-79 years (33\%) (Table 3). The distribution for sex in the experimental group, as in the total and experimental groups, is constituted by a prevalence of women with a ratio of $2: 1$.

The results of the balance tests show an improvement in $50 \%$ of the cases ( 9 out of 18); stability in $50 \%$ of cases (9 out of 18) and no cases of deterioration. Data from the gait test show an improvement in $27.77 \%$ (5 out of 18 ), a stability in
$55.55 \%$ (10 out of 18) of the cases and worsened in $16.6 \%$ of cases ( 3 out of 18 ). In the sit-to-stand test, an improvement was registered in $38.8 \%$ (7 out of 18); that related to stability is $55.5 \%$ (10 out of 18 ); while the inherent deterioration is $5.5 \%$ ( 1 out of 18 ).

Across the total values, an improvement was observed in $72.2 \%$ (13 out of 18) of the cases, in $16.6 \%$ ( 3 out of 18) of the cases the values were stable and in $11.1 \%$ ( 2 out of 18 ) conditions had worsened (Table 4).

Fig. 1 shows the changes in SPPB scores in the experimental group who followed the APA protocol. 


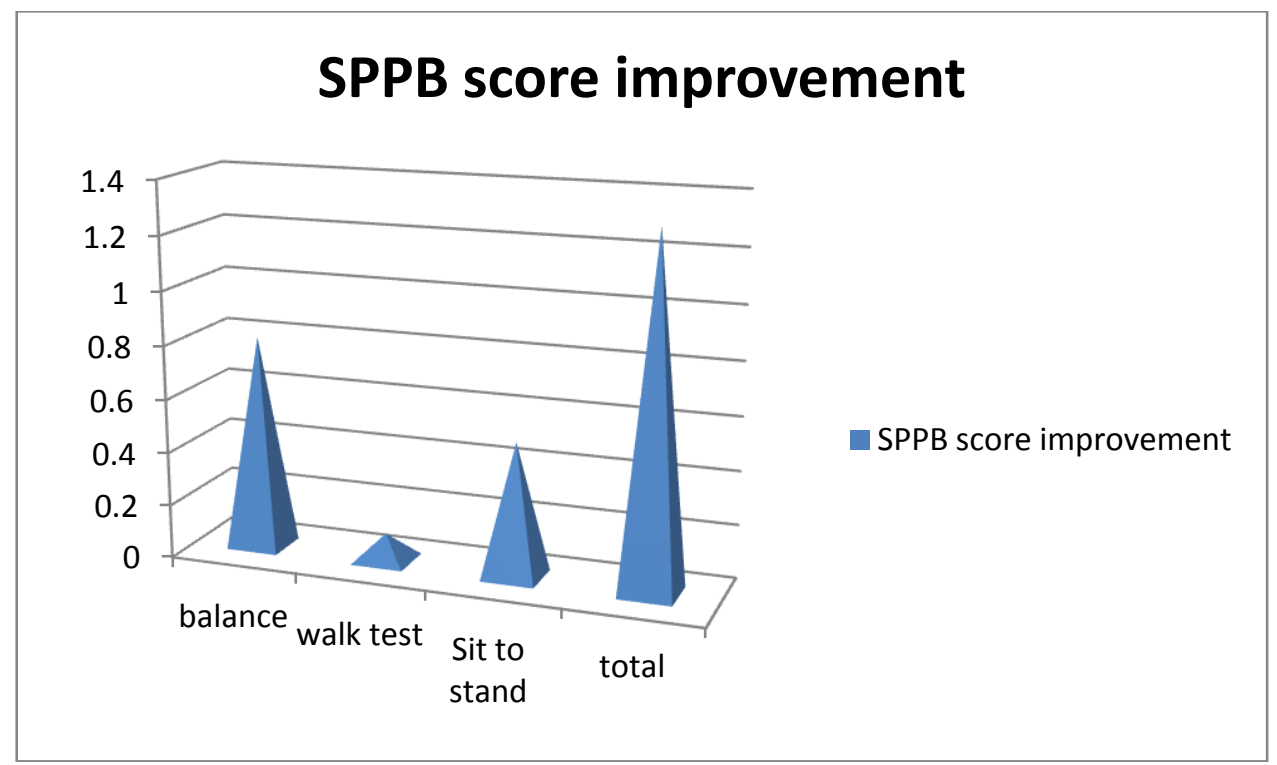

Fig. 1 Changes (improvement) in SPPB score in the experimental group.

Table 6 Changes in SPPB score from baseline classed into three categories (improvement, unchanged and worsening) in control group.

\begin{tabular}{lllll}
\hline Changes & Balance & Walk test & Sit to stand & Total \\
\hline Improvement & $20 \%$ & $6 \%$ & $33.3 \%$ & $33.3 \%$ \\
Unchanged & $60 \%$ & $66.6 \%$ & $40 \%$ & $33.3 \%$ \\
Worsening & $20 \%$ & $27.6 \%$ & $26.6 \%$ & $33.3 \%$ \\
\hline
\end{tabular}

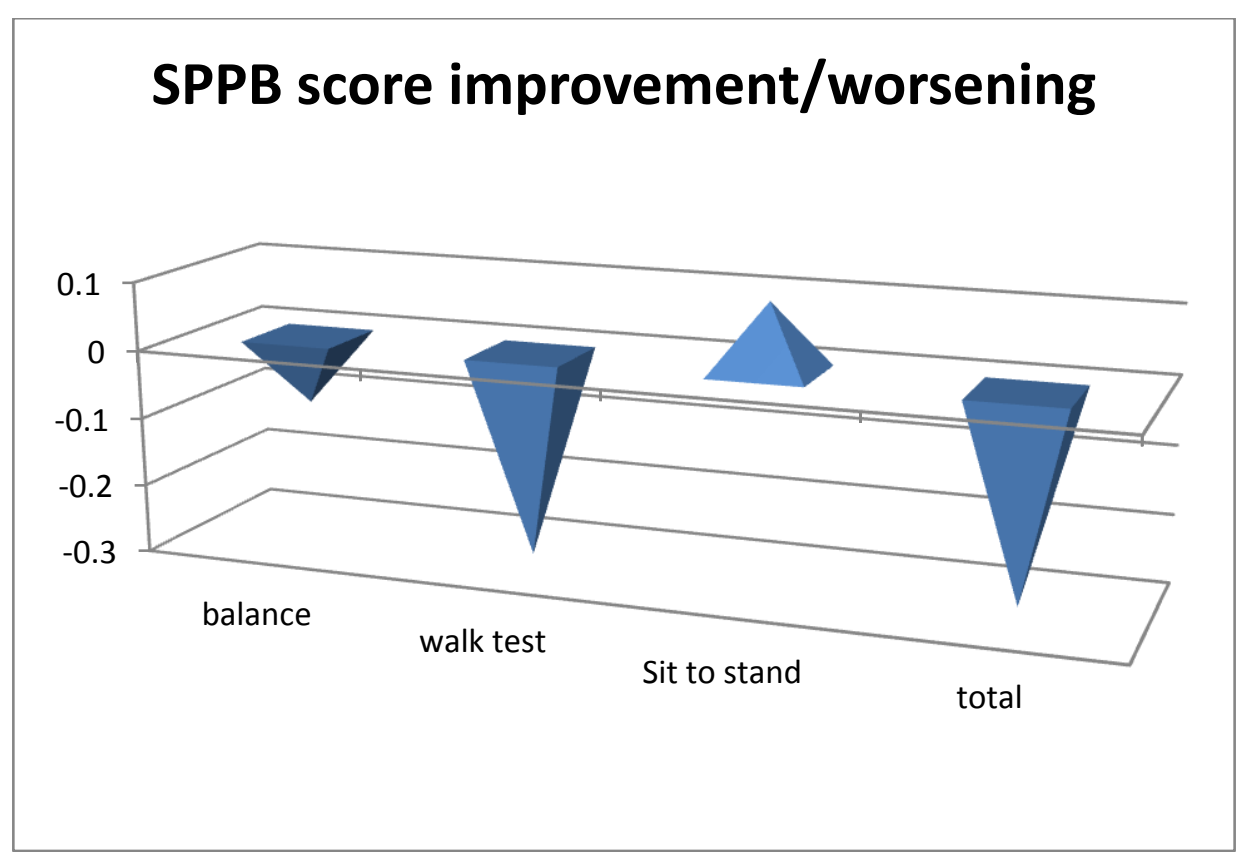

Fig. 2 Changes (improvement/worsening) in SPPB score in the control group.

The data presented includes the average differences between the pre and post tests for the three separate tests (balance, gait and sit-to-stand) and their sum. In the experimental group, the result related to the balance sub-test shows a final improvement of 0.8. With reference to the gait test, a final improvement of 0.1 
was recorded. For the sit-to-stand, the average of the input values shows an improvement of 0.5. In relation to the total of the values obtained for each item, an improvement of 1.3 was recorded.

In the control group, the results of the balance test show an improvement in $20 \%$ of the cases ( 3 out of 15 ); stability in $60 \%$ of cases ( 9 out of 15) and worsening in $20 \%$ (3 out of 15). Data from the gait test show an improvement in $6 \%$ of the cases ( 1 out of 15), stability in $66.6 \%$ (10 out of 15 ) of the cases and worsening in
$27.7 \%$ of cases ( 4 out of 15 ). In the sit-to-stand test, an improvement was encountered in $33.3 \%$ (5 out of 15 ) of the cases; related to the stability is $40 \%$ (6 out of 15); that concerning the deterioration is $26.6 \%$ ( 4 out of 15 ). The total values show that in $33.3 \%$ (5 out of 15) of the cases an improvement occurred; in 33.3\% (5 out of 15) of the cases the values were stable and in $33.3 \%$ (5 out of 15) a worsening of the conditions was encountered (Table 6).

In the control group, the average incoming results

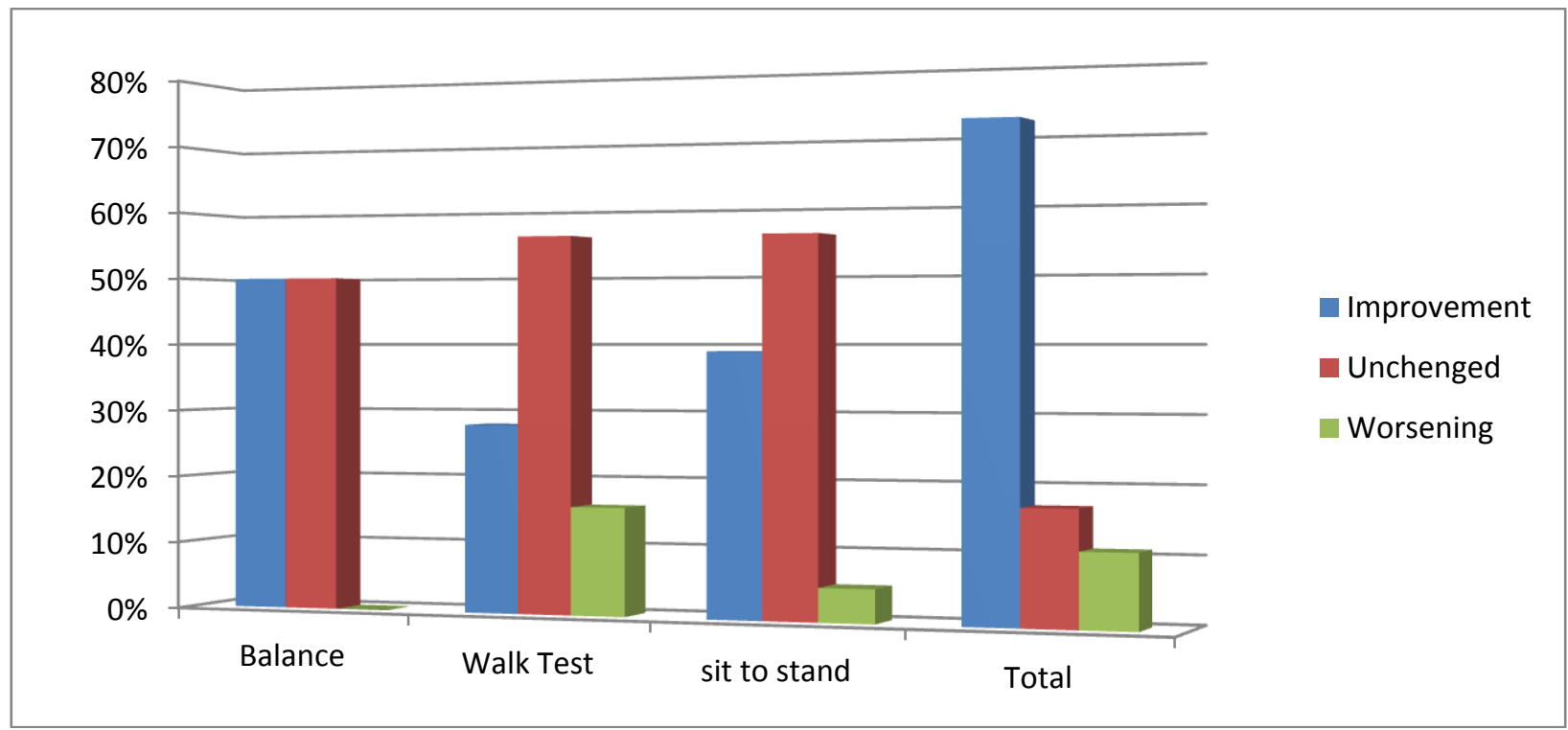

Fig. 3 SPPB score classed into three categories (improvement, unchanged and worsening) in experimental group.

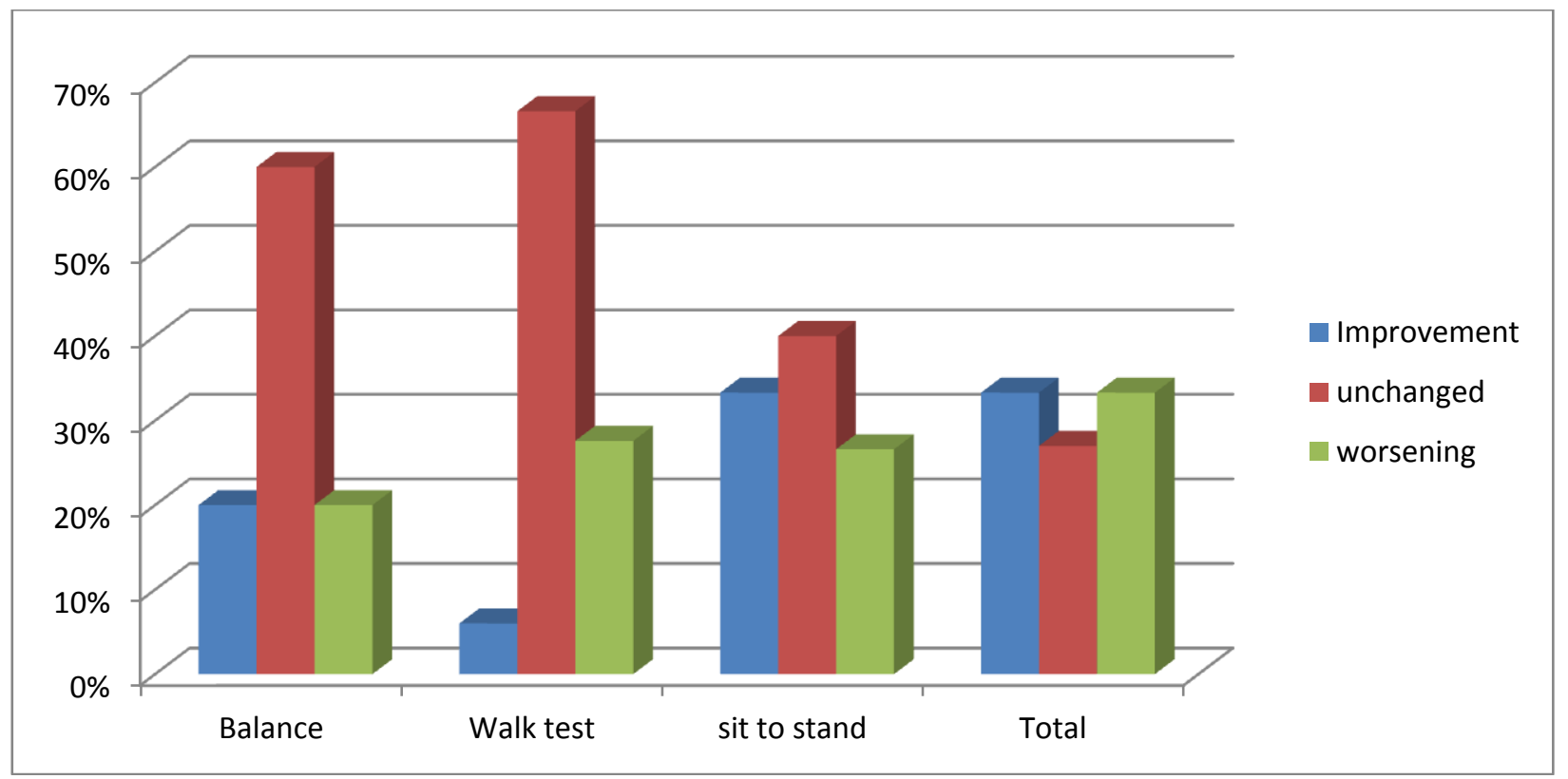

Fig. 4 SPPB score classed into three categories (improvement, unchanged and worsening) in control group. 


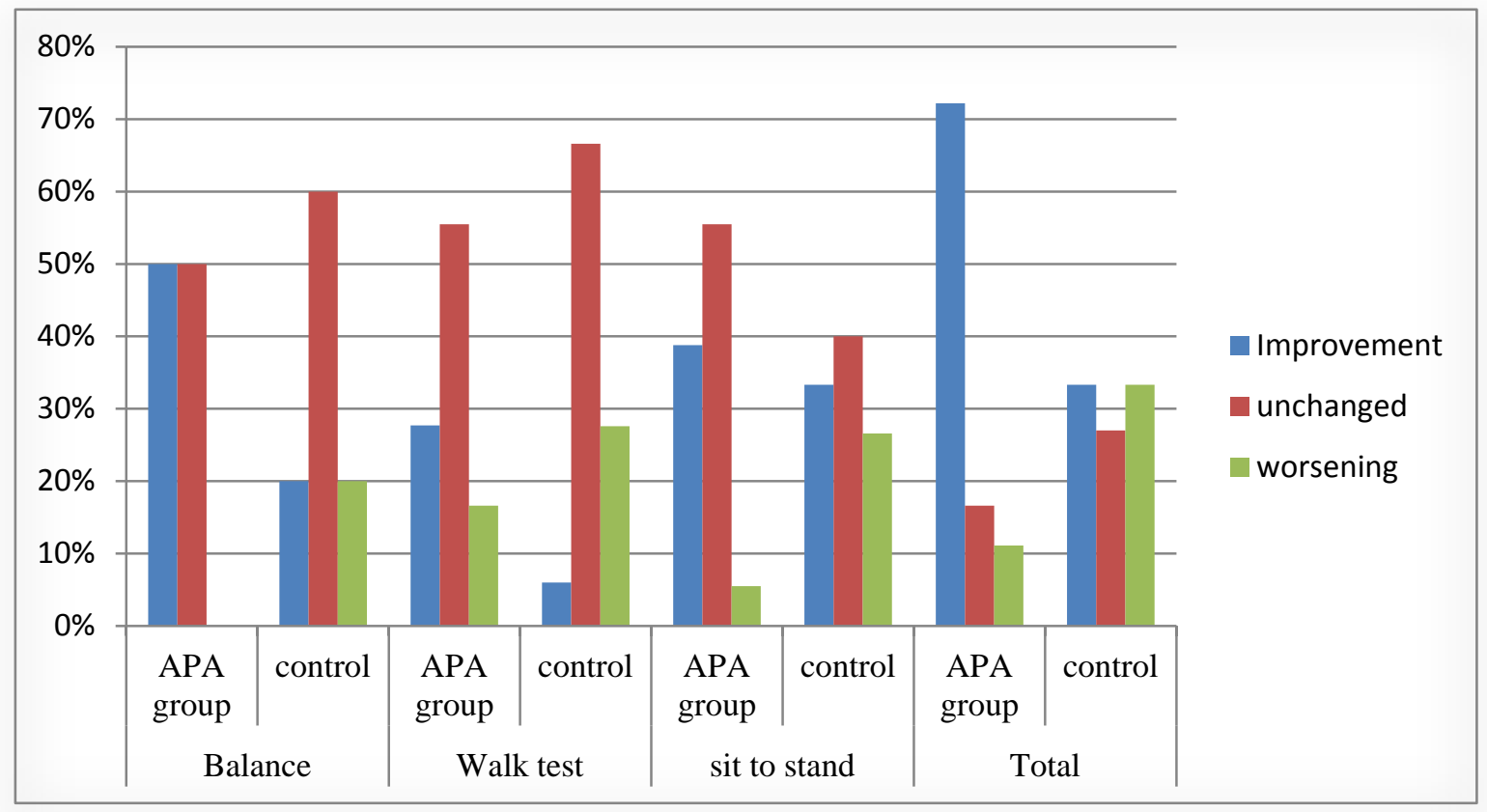

Fig. 5 Comparison of SPPB score classified into three categories (improvement, unchanged and worsening) between experimental group and control group.

related to the balance sub-test shows a final worsening of 0.1 . The average value, with reference to the gait test input in the experimental group obtained a final worsening of 0.3. At the sit-to-stand, the average test values show an improvement of 0.1 . In relation to the total of the values obtained for each item, a worsening of 0.3 was registered (Fig. 2).

\section{Discussions and Conclusions}

The aim of this study was to evaluate possible improvements and/or consolidations of motor performance, in particular gait speed, balance, and the strength of the lower limbs, as a result of the implementation of structured APA (adapted physical activity).

The effects of the APA protocol was measured using the SPPB (Short Physical Performance Battery) before and after the 18-week intervention, which included two weekly sessions of structured training modules focusing on the coordinating and organic conditioning of the elderly.

From the results obtained, a substantial improvement in elderly physical performance, investigated via SPPB, was found following a predictable and structured adapted physical activity protocol (Fig. 3).

From the comparison of the data, in fact, there is a substantial improvement in motor performance investigated with SPPB in the experimental group compared to the control group (72.2\% vs. 33.3\%) (Figs 4 and 5).

These data lend support to previous studies that have reported improvement, maintenance and recovery of motor performance of the elderly as a result of the implementation of structured adapted physical activity $[17,18,26-28]$.

The results of this study lead us to reflect on the importance of implementing structured adapted physical activity in elderly people centers as an instrument of promoting the maintenance and development of autonomy and well being among the elderly.

This study also provides significant implications for the analysis of instruments used for the assessment and evaluation of physical performance of the elderly 
SPPB with particular reference to the effects of an APA intervention. Studies on the assessment of motor activity are an essential element for effective teaching of movement. In fact, analysis of results based on standardized assessment tests provides the possibility to base the teaching of the movement on the principle of personalization. This is essential since personalized teaching enhances individuals' capacity and potential by taking into consideration the individuals' specificities and difficulties.

\section{References}

[1] Melton, L. J., Khosla, S., Crowson, C. S., O’Connor, M. K., O'Fallon, W. M., and Riggs, B. L. 2000. "Epidemiology of Sarcopenia." Journal of the American Geriatrics Society 48 (1): 25-30.

[2] Hughes, V. A., Frontera, W. R., Wood, M., Evans, W. J., Dallal, G. E., Roubenoff, R., and Singh, M. A. F. 2001. "Longitudinal Muscle Strength Changes in Older Adults: Influence of Muscle Mass, Physical Activity, and Health." Journals of Gerontology 56 (5): 209-17.

[3] Clemson, L., Fiatarone Singh, M. A., Bundy, A., Cumming, R. G., Manollaras, K., O’Loughlin, P., and Black, D. 2012. "Integration of Balance and Strength Training into Daily Life Activity to Reduce Rate of Falls in Older People (the LiFE study): Randomised Parallel Trial." British Medical Journal 345: e4547.

[4] European Commission 2013. "Horizon 2020. European Commission Decision." Work Program 2014-2015 C 8631 of 10 December 2013.

[5] World Health Organization 2012. "Global Brief for World Health Day 2012: Good Health Adds Life to Years." Geneva, Switzerland: WHO Document Production Services. Accessed August 5, 2015. http://www.who.int/world_health_day/2012

[6] Macchi, C. 2007. Motor Activity, Fitness and Health and the Elderly. Firenze: SEE, 65-143. (in Italian)

[7] Sun, F., Norman, I. J., and While, A. E. 2013. "Physical Activity in Older People: A Systematic Review." BMC Public Health 13 (1): 449.

[8] D'Elia, F., Vastola R., and Sibilio, M. 2014. "The Use of Exergames for Adaptive Physical Activity among the Elderly." Journal of Sports Science 2: 47-52.

[9] Candela, F., Liubicich, E. M., Di Marco, M., Magistro, D., Nebiolo, S., and Rabaglietti, E. 2010. "Effects of Physical Activity on the Emotional Tone and the Perception of Limitation of Health for Everyday Activities in Institutionalized Elderly: A Longitudinal Study." In IX National Congress SIPSA. Health Psychology between
Research and Intervention in Health and Social Services in the Community, in the Workplace and in School. Bergamo: Franco Angeli. (in Italian)

[10] Littbrand, H., Lundin, O. L., Gustafson, Y., and Rosendahl, E. 2009. "The Effect of a High Intensity Functional Exercise Program on Activities of Daily Living: A Randomized Controlled Trial in Residential Care Facilities." Journal of the American Geriatrics Society 57 (10): 1741-9.

[11] Bean, J. F., Vora, A., and Frontera, W. R. 2004. "Benefits of Exercise for Community-Dwelling Older Adults." Archives of Physical Medicine and Rehabilitation 85 (3): 31-42.

[12] SSAI. 2013. "Census of Facilities for the Elderly." Interior Ministry School Administration of the Interior. The Notebooks of Documentation N.2/2013. (in Italian)

[13] Bird, M., Hill, K. D., Ball, M., Hetherington, S., and Williams, A. D. 2011. "The Long-Term Benefits of a multi-Component Exercise Intervention to Balance and Mobility in Healthy Older Adults." Archives of Physical Medicine and Rehabilitation 52 (2): 211-6.

[14] Whitehurst, M. A., Johnson, B. L., Parker, C. M., Brown, L. E., and Ford, A. M. 2005. "The Benefits of a Functional Exercise Circuit for Older Adults." Journal of Strength and Conditioning Research 19 (3): 647-51.

[15] Giné-Garriga, M., Guerra, M., Pagès, E., Manini, T. M., Jiménez, R., and Unnithan, V. B. 2010. "The Effect of Functional Circuit Training on Physical Frailty in Frail Older Adults: A Randomized Controlled Trial.” Journal of Aging Physical Activity 18 (4): 401-24.

[16] Mazzeo, R. S., Cavanagh, P., Evans, W. J., Fiatarone, M., Hagberg, J., McAuley, E., and Startzell, J. 1998. "Exercise and Physical Activity for Older Adults." American College of Sports Medicine Position Stand. MedSci. Sports Exerc. 330 (25): 1769-75.

[17] Benvenuti, F., Nucida, V., Taviani, A., and Macchi, C. 2012. "Definition and Purpose of the APA: Didactic Implications." In Methodology and Teaching Adapted Physical Activity (APA), edited by Macchi, C., and Benvenuti, F. Firenze: Master Books, 19-22. (in Italian)

[18] Macchi, C. 2012. Theory and Technique of Physical Activity Adult-Elder. Firenze: Master Books: 15-40. (in Italian)

[19] Liu. C. J., and Latham, N. 2011. "Can Progressive Resistance Strength Training Reduce Physical Disability in Older Adults? A Meta-analysis Study." Disability and Rehabilitation 33 (2): 87-97.

[20] Guralnik, J. M., Ferrucci, L., Pieper, C. F., Leveille, S. G., Markides, K. S., Ostir, G. V., Studenski, S., Berkman, L.F., and Wallace, R. B. 2000. "Lower Extremity Function and Subsequent Disability Consistency across Studies, Predictive Models, and Value of Gait Speed Alone 
Compared with the Short Physical Performance Battery." The Journals of Gerontology Series A: Biological Sciences and Medical Sciences 55 (4): 221-31.

[21] Guralnik, J. M., Simonsick, E. M., Ferrucci, L., Glynn, R. J., Berkman, L. F., Blazer, D. G., Scherr, P. A., and Wallace, R. B. 1994. "A Short Physical Performance Battery Assessing Lower Extremity Function: Association with Self-reported Disability and Prediction of Mortality and Nursing Home Admission." Journals of Gerontology 49 (2): 85-94.

[22] Volpato, S., Cavalieri, M., Sioulis, F., Guerra, G., Maraldi, C., Zuliani, G., Fellin, R., and Guralnik, J. M. 2011. "Predictive Value of the Short Physical Performance Battery Following Hospitalization in Older Patients." $J$. Gerontol. 66 (1): 89-96.

[23] Gawel, J., Vengrow, D., Collins, J., Brown, S., Buchanan, A., and Cook, C. 2012. "The Short Physical Performance Battery as a Predictor for Long Term Disability or Institutionalization in the Community Dwelling Population Aged 65 Years Old or Older." Physical
Therapy Review 17 (1): 37-44.

[24] Choi, H. C., Son, K. Y., Cho, B., Park, S. M., and Cho, S. I. 2012. "An Implication of the Short Physical Performance Battery (SPPB) as a Predictor of Abnormal Pulmonary Function in Aging People." Archives of Gerontology and Geriatrics 54 (3): 448-52.

[25] Cerullo, F., Colloca, G., Ferrini, A., Ciaburri, M., Scambia, G., Bernabei, R., Gambassi, G., and Cesari, M. 2011. "Measurements of Physical Function in Geriatrics." $G$. Gerontol. 59: 265-72. (in Italian)

[26] Warburton, D. E., Nicol, C. W., and Bredin, S. S. 2006. "Health Benefits of Physical Activity: The Evidence." Canadian Medical Association Journal 174 (6): 801-9.

[27] Chodzko-Zajko, W. J. 2014. "Exercise and Physical Activity for Older Adults." Human Kinetic 3 (1): 101-6.

[28] Gillies, E., Aitchison, T., MacDonald, J., and Grant, S. 1999. "Outcomes of a 12-Week Functional Exercise Programme for Institutionalised Elderly People." Physiotherapy 85 (7): 349-57. 